\title{
Power transformer fault diagnosis system based on Internet of Things
}

\author{
Guoshi Wang ${ }^{1 *}$, Ying Liu², Xiaowen Chen ${ }^{1}$, Qing Yan ${ }^{1}$, Haibin Sui ${ }^{1}$, Chao Ma ${ }^{1}$ and Junfei Zhang ${ }^{1}$
}

\section{*Correspondence:}

hello123381@163.com

1 Hainan Power Grid Co.,

Ltd, Haikou 460100, Hainan,

China

Full list of author information is available at the end of the article

\begin{abstract}
Transformer is the most important equipment in the power system. The research and development of fault diagnosis technology for Internet of Things equipment can effectively detect the operation status of equipment and eliminate hidden faults in time, which is conducive to reducing the incidence of accidents and improving people's life safety index.
\end{abstract}

Objective: To explore the utility of Internet of Things in power transformer fault diagnosis system.

Methods: A total of 30 groups of transformer fault samples were selected, and 10 groups were randomly selected for network training, and the rest samples were used for testing. The matter-element extension mathematical model of power transformer fault diagnosis was established, and the correlation function was improved according to the characteristics of three ratio method. Each group of power transformer was diagnosed for four months continuously, and the monitoring data and diagnosis were recorded and analyzed result. GPRS communication network is used to complete the communication between data acquisition terminal and monitoring terminal. According to the parameters of the database, the working state of the equipment is set, and various sensors are controlled by the instrument driver module to complete the diagnosis of transformer fault system.

Results: The detection success rate of the power transformer fault diagnosis system model established in this paper is as high as 95.6\%, the training error is less than 0.0001, and it can correctly identify the fault types of the non training samples. It can be seen that the technical support of the Internet of Things is helpful to the upgrading and maintenance of the power transformer fault diagnosis system.

Keywords: Internet of things, Power transformer, Fault diagnosis, Wireless communication and network

\section{Introduction}

In the power supply system, transformer plays an important role in power production and transmission. If the transformer breaks down suddenly in the process of operation, it will cause serious harm to human safety and economic property. Therefore, it is of great value to improve the specific monitoring and troubleshooting of transformers.

The application of the Internet of Things to the power system can not only improve the basic network of power transmission and communication, but also enhance the security and stability of its transmission and communication network. As the main author(s) and the source, provide a link to the Creative Commons licence, and indicate if changes were made. The images or other third party material in this article are included in the article's Creative Commons licence, unless indicated otherwise in a credit line to the material. If material is not included in the article's Creative Commons licence and your intended use is not permitted by statutory regulation or exceeds the permitted use, you will need to obtain permission directly from the copyright holder. To view a copy of this licence, visit http:// creativecommons.org/licenses/by/4.0/. 
communication channel of distribution network, it can provide the functions that the current public and wireless networks lack. In addition, the Internet of Things can withstand the power emergency in negative weather and improve the disaster resistance ability of the communication system. The Internet of Things will become an important way for the rapid development of information technology in the future.

Di Renzo's research relied on modeling the location of the base station as the points of spatial Poisson point process, and carries out system level analysis by using random geometry. The trade-off between wireless information and power transmission is described by the concept of "feasible region," and quantified by the joint cumulative distribution function of average power and average rate. Di Renzo proposed a new mathematical method to analyze and optimize cellular low-energy mobile devices with wireless information and power transmission capabilities. The method is ingenious, but requires high accuracy, and needs to be improved in practical application [1]. In order to reduce the difficulty of data acquisition and transmission in the fault diagnosis system of mine hoist, and solve the problems of low efficiency of fault diagnosis and unreasonable reasoning process of mine hoist, a fault diagnosis method of mine hoist equipment based on Internet of Things is proposed. Based on ZigBee short-range wireless communication technology, the collaborative acquisition system of key components of mine hoisting equipment is designed in the Internet of Things. Juanli adopted remote wireless general packet radio service (GPRS) transmission mode to realize real-time data acquisition and network layer establishment. Juanli through the fault diagnosis test of mine hoist, the diagnosis method obtains complete diagnosis data, and the diagnosis result of this method has high accuracy and reliability [2]. Qian designed a relay with dual interface of wireless and PLC, which connects PLC and wireless sensor into an Internet of Things network. In addition, the dual interface relay adaptively selects the interface to forward the message according to the channel state. Qian proposed a general mathematical model of the dual interface relay protection system. According to the statistical characteristics of PLC and wireless channel, Qian derived the probability density function of output signal-to-noise ratio (SNR). Qian's research has constructed the general mathematical model of double interface relay protection system. This method has high accuracy, but lacks feasibility [3]. Zikria connected the digital and physical worlds by combining energy-efficient microcontrollers, low-power transceivers, sensors, and actuators in so-called "small objects." In general, intelligent objects are severely limited in computing, memory and energy resources. New verification methods and experimental tools are needed to study the intelligent object network, new software platform is needed to operate intelligent objects effectively, and innovative network models and protocols are needed to interconnect intelligent objects. Zikria's research connected the digital world with the physical world, which has many innovations, but needs more verification methods to support the accuracy of the research data [4].

In this paper, a total of 30 groups of transformer fault samples are selected, and 10 groups are randomly selected for network training, and the remaining samples are used for testing. The transformer fault diagnosis system model is established for each group of power transformers for four consecutive months, and the monitoring data and diagnosis results are recorded and analyzed. The communication network uses GPRS to realize the communication between the data acquisition terminal and the monitoring terminal. 
According to the parameters of the sample library, the working state of the equipment is set, and various sensors are controlled by the instrument driver module to complete the detection of the transformer fault system. The results show that the detection success rate of the power transformer fault diagnosis using this model is as high as 95.6, and the training error is less than 0.0001 , and it can accurately identify the fault types of the samples in the non training set.

\section{Principle about Internet of Things and fault diagnosis system}

\subsection{Internet of Things}

\section{Combination of Internet of Things technology and smart grid}

Since its successful development, the Internet of Things has undergone many significant changes. From the logistics network mainly relying on radio frequency identification technology at the beginning, it has now been studied that a variety of information sensing equipment relying on Internet technology, sensing technology and computer technology can use Internet facilities for information exchange, coordination and processing. Then, it is an interconnection to meet the requirements of information exchange between people and objects in various regions or large areas $[5,6]$. The application of Internet of Things technology in smart grid can realize the perception and exchange of advanced level, convenient operation and unified standard communication information, and complete the distributed intelligent information transmission, measurement and control. Using smart sensors to connect a variety of equipment and facilities as a whole, and then constitute an integrated information service system, not only can process and analyze a variety of information, but also can save costs through this way, and optimize the operation and control of power grid.

\section{The role of IOT technology in power maintenance}

The periodic maintenance mode formulated in 1950s is used for the maintenance of power equipment in China. So far, the main maintenance mode is still the method $[7,8]$. There are two inevitable deficiencies in this method. One is the large-scale maintenance of normal equipment, the other is the equipment damage caused by accidents, which forces the regular maintenance to inevitably lead to a large amount of human and material resources consumption.

The primary problem to be solved by Internet of Things technology is information acquisition. How to process information and how to realize overhaul decision should be scientifically planned $[9,10]$. As an independent management subject, maintenance management is inseparable from the advanced technology management system. A variety of maintenance systems are composed of various management objectives in many countries around the world. Under the condition of market factors and technical level, a maintenance management mechanism including conditional maintenance and flexible application of various methods is proposed. The addition of diagnosis expert system can make the reliability and safety of the system meet the level of people's needs, and optimize the maintenance plan and maintenance process. 


\section{Power transformer}

\section{Brief introduction}

Power transformer is one of the key equipment of power system, and its reliable operation characteristic is the guarantee of the whole power mechanism to supply power safely. The fault of power transformer will directly interfere with the normal operation of the power system, and also cause serious harm to the economy of power companies and power users [11]. At present, the power system is developing rapidly in the direction of ultra-high voltage, large power grid and automation, and the influence and interference of transformer fault on the normal operation of power system is becoming more and more serious [12]. Because the improvement process of power transformer fault is related to the operating environment, load capacity and insulation level, it is difficult to find out some hidden faults immediately by using preventive maintenance means. Preventive maintenance not only needs high cost, but also does not have advanced level for development faults such as insulation. Therefore, it is very useful to detect the transformer running fault system on-line at any time. The combination of on-line detection technology and intelligent diagnosis of transformer fault can timely discover the hidden dangers in the development, reduce the probability of accidents, and do a good job of prevention.

\section{Application of power transformer}

In the contemporary society, electricity is a particularly important energy, it has penetrated into every corner of human society. However, the power generated by power plants usually needs to be transported over long distances to reach the power area $[13,14]$. When the transmission power is fixed, because the voltage and current are in positive proportion, the current required for transmission decreases with the increase in voltage. Because the impedance of the transmission path is almost a fixed value, and the line loss is proportional to the square of the current, increasing the transmission voltage can greatly reduce the line voltage drop and line loss. At present, there are great difficulties in the manufacture of high-voltage generators. Therefore, special equipment must be used to boost the voltage before the voltage at the end of the generator is sent out. This special equipment is the transformer. In addition, at the receiving end, it is necessary to use step-down transformer to reduce the high-voltage to the distribution system voltage, so many distribution transformers are used to regulate the high-voltage to the exact application value. In the power system, transformers occupy a very important position, not only need a large number, but also have high efficiency and stable operation.

When the transformer is in operation, the transformer will fail due to various factors $[15,16]$. If the transformer fails, it will affect the output of the generator, reduce and interrupt the power supply of some users, and prolong the maintenance time of the transformer. If the accident cannot be detected and solved immediately, it will cause serious harm to the conventional power supply and residents' property. 


\subsection{Power transformer fault diagnosis system based on Internet of Things}

The decision-level fusion in the system uses extension theory to establish a fault diagnosis model, uses correlation functions for evaluation, and comprehensively diagnoses the fault type of the transformer $[17,18]$. The specific implementation process of decision-level data fusion based on extension theory is as follows:

\section{Structure matter element matrix}

According to the concept of matter element and the ratio coding of each transformer and the fault type, the transformer fault type is divided into 9 levels, namely: $U=\{u 1$, $u 2, u 4, u 5, u 6, u 7, u 8, u 9\}=\{I 1, I 2, I 4, I 5, I 6, I 7, I 8, I 9\}$, and according to the three ratio codes of $\mathrm{C}_{2} \mathrm{H}_{2} / \mathrm{C}_{2} \mathrm{H}_{4}, \mathrm{CH}_{4} / \mathrm{H}_{2}, \mathrm{C}_{2} \mathrm{H}_{4} / \mathrm{C}_{2} \mathrm{H}_{6}$ and the corresponding failure interval, the three-dimensional matter element matrix constructed is:

$$
\begin{aligned}
& Z=\left[\begin{array}{ccc}
\text { Transformer } & \text { Ratio of } \mathrm{C}_{2} \mathrm{H}_{2} / \mathrm{C}_{2} \mathrm{H}_{4} & \text { Quantities of Failure Types } V_{1} \\
\text { Fault Type } & \text { Ratio of } \mathrm{CH}_{4} / \mathrm{H}_{2} & \text { Quantities of Failure Types } V_{2} \\
U & \text { Ratio of } \mathrm{C}_{2} \mathrm{H}_{4} / \mathrm{C}_{2} \mathrm{H}_{6} & \text { Quantities of Failure Types } V_{3}
\end{array}\right] \\
& \text { BMI }(b)=2 n \ln (\sigma)+n \ln (2 \pi)+n\left\{\frac{n+\operatorname{tr}(S)}{n-2-\operatorname{tr}(S)}\right\}
\end{aligned}
$$

The classic domain-free matrix for determining the fault type is:

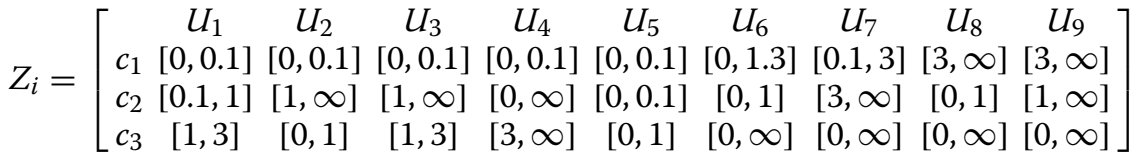

$$
\begin{aligned}
& W(T)=K(y(T-1), \ldots, y(t-n), u(T-d-1), \ldots, u(k-d-n))
\end{aligned}
$$

The analysis result of the detected dissolved gas in the transformer oil is represented by the matter element $\mathrm{ZO}$, and the following three-dimensional matrix of the matter to be diagnosed is obtained:

2. Determine the substance to be diagnosed

$$
Z_{0}=\left[\begin{array}{lll}
U_{0} & c_{1} & V_{1} \\
& c_{2} & V_{2} \\
& c_{3} & V_{3}
\end{array}\right]
$$

3. For the status information of each group of data, the steps to determine the objective weight of the status information between components by the Ou method are as follows: 


$$
\begin{aligned}
& C(k)=\left[\zeta_{1} c_{1}(t)+\zeta_{2} c_{2}(k)+\zeta_{3} c_{3}(k)+\zeta_{4} c_{4}(k)+\zeta_{5} c_{5}(k)+\zeta_{6} w_{i k}\right] \\
& c_{1}(t) \geq 0, c_{2}(k) \geq 0, c_{3}(k) \geq 0, c_{4}(k) \geq 0, c_{5}(k) \geq 0 \\
& p_{1}^{*}=\frac{2 k}{k+1}+\frac{2 c_{1}+c_{2}+3 e t+2 e t \zeta}{3} \\
& I=\frac{n \sum_{i=1}^{n} \sum_{j=1}^{n} w_{i_{j}}\left(x_{i}-\bar{x}\right)(x j-\bar{x})}{\sum_{i=1}^{n} \sum_{j=1}^{n} w_{i j}(x i-\bar{x})^{2}}=\frac{n \sum_{i=1}^{n} \sum_{i \neq j}^{n} w_{i_{j}}\left(x_{i}-\bar{x}\right)(x j-\bar{x})}{S^{2} \sum_{i=1}^{n} \sum_{j=1}^{n} w_{i j}}
\end{aligned}
$$

1. Calculate the ratio of the state information value $x_{i j}$ under the state information $P_{i j}$ amount $j$ :

$$
\begin{aligned}
& P_{i j}=\frac{x_{i j}}{\sum_{i=1}^{m} x_{i j}} \\
& W(T)=K(y(T-1), u(T-d-1)) \\
& v=Q\left(D_{2}\right) 2 \times \pi \times 3600 \\
& U=\sum_{i=1}^{g}\left\{P_{i} \mid \sum_{j=1}^{k} p_{j}^{(i)}\right\}
\end{aligned}
$$

2. Calculate the value of state information $j$ :

$$
\begin{aligned}
& h_{j}=-k \sum_{i=1}^{m}\left(p_{i j} \ln p_{i j}\right) \\
& U=\left\{P_{1}\left|D, L, f_{2}, Q, d, l \quad P_{2}\right| f_{1}, \mu \quad P_{3} \mid N, M, I\right\} \\
& I R=\sum_{S-1}^{U} \sum_{d-1}^{K} f_{s}, D V_{s}, d
\end{aligned}
$$

Among them, $k=\frac{1}{\ln (m)}, k>1$, and $0 \leq h_{j} \leq 1$.

3. Calculate the difference coefficient of state information $j g_{i}$ : 


$$
\begin{aligned}
& g_{i}=1-h_{j}\left(0 \leq h_{j} \leq 1\right) \\
& \beta_{j}=\left(X^{T} W_{j} X\right)^{-1} X^{T} W_{j} Y
\end{aligned}
$$

\section{Data collection terminal}

\section{GPRS communication module}

The online monitoring and diagnosis system will use the GR47 module in the embedded TCP/IP protocol stack. The GR47 module mainly includes GPRS communication module and IP module, which are used to complete the TCP/IP protocol replacement, complete the receiving and sending of various information in turn, and provide data supply for GPRS $[19,20]$. In order to achieve various types of operating states, this module has also designed various types of interfaces to meet work requirements. The adoption of this module provides a lot of convenience for many users. It is mainly reflected in the fact that the protocol replacement process between TCP and IP does not need to send people to manually operate, which reduces a variety of complicated control time, and makes data transmission more clear and active. The transmitted data can reach the terminal of the other party's network system directly after GR47 processing, which greatly reduces the development time of the system.

\section{Fault diagnosis module}

After receiving the exact data information, the fault diagnosis module is fused and analyzed, and compared with the sample parameters in the diagnosis database to determine whether the transformer is intact and its failure mechanism [21, 22]. If the fault is a general type of low function, the diagnostic system will give a warning, allowing the user to complete the simple equipment adjustment according to the given operation steps [23]. When the fault is more serious and the performance is worse, the diagnostic system will compare the collected data with the corresponding parameters of the transformer, analyze the self-check data and important information in the technical reference library in all aspects, and determine the modules that can be replaced after the fault occurs. After meeting the previous requirements, the system will give the user the specified repair steps. In this stage, the system technical guidance from the determination of the transformer fault type to the fault resolution method is completed.

\section{Transformer fault diagnosis based on information fusion technology}

Due to the complexity of the transformer manufacturing process and the instability of the operating state, the variety of transformer fault causes is caused, which is mainly reflected in the same type of fault mode with different fault characteristics, and the same type of fault characteristic is the combined result of multiple failure modes [24, 25]. Therefore, there are various nonlinear correspondences between failure modes and failure characteristics. Therefore, in the transformer fault diagnosis, determining its 
relationship is extremely critical. Information fusion technology is to analyze and process information and data from various information sources, to maximize the use of all fault characteristics of the transformer, to obtain multiple aspects of the same object of the transformer from multiple angles, and to integrate them to achieve better accurate and more scientific online diagnosis. After the initial data evaluation and simple fault diagnosis and analysis, the final result needs to be displayed concisely and clearly on the LCD screen of the monitoring site to facilitate the direct collection of information and management planning.

\section{Experiment and analysis}

\subsection{Materials and experimental design}

1. Data collection and determination of training samples

Select 30 groups of transformer fault samples, and randomly select 10 groups for network training. The remaining samples are used for testing. According to the designed transformer diagnosis system model, each group of power transformers will be diagnosed for four consecutive months, and the monitoring data will be recorded and analyzed.

2. Information analysis and processing (flowchart)

The GPRS communication network mode is used to complete the communication between the data acquisition terminal and the monitoring terminal. The data acquisition terminal transmits the data of the detected transformer parameters to the monitoring terminal or receives the monitoring information of the monitoring terminal. The flowchart of the inspection plan is shown in Fig. 1.

3. Detection and fault diagnosis module Measure the dissolved gas in the transformer oil and cooperate with the monitoring management system, compare with the standard parameters in the diagnostic knowledge base, determine the performance status and fault type of the voltage transformer, give the user the operation steps for maintenance, and finally complete the fault type analysis of the transformer, and provide data support to technicians.

4. Status monitoring module and IoT management system

Set the operating status of the equipment according to the database parameters, and use the instrument driver module to control multiple sensors to complete the monitoring of the transformer operating status, and take appropriate measures to protect and control the transformer.

5. Calculation formulas needed by each module in the experiment

1. Calculate the correlation between the object to be measured and the standard faulty object: 


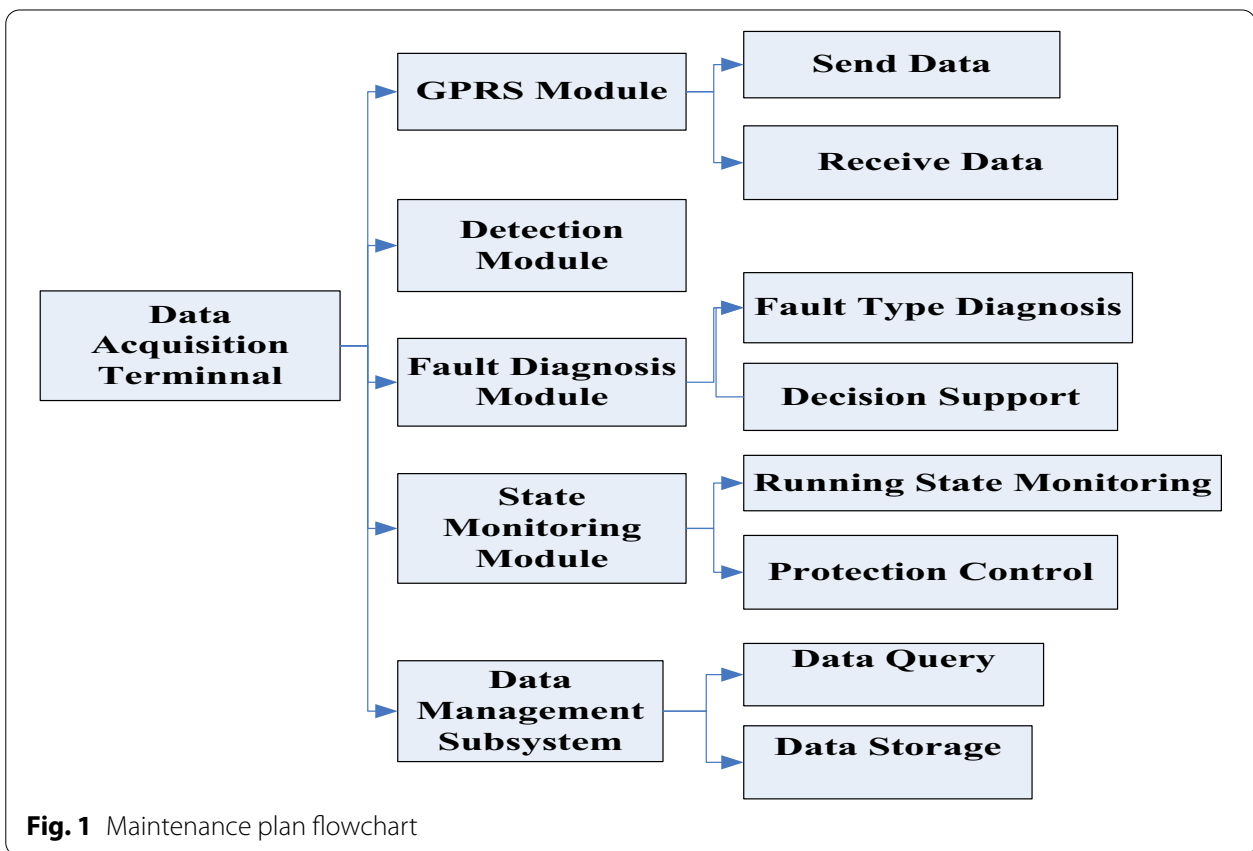

$$
\begin{aligned}
& K\left(N_{i}\right)=\sum_{j=1}^{3} w_{j} \cdot K\left(v_{j}\right) \\
& \sigma_{i k j l}= \begin{cases}\frac{n}{\Delta_{i k j l}} \sqrt{\sum_{s=1}^{n}\left(x_{i k}(\varepsilon)-x_{j l}(\varepsilon)\right)^{2} \Delta_{i k j l}(\varepsilon)} & \Delta_{i k j l}>0 ; \\
0 & \Delta_{i k j l}<0\end{cases}
\end{aligned}
$$

Among them $w_{j}$ is the weight coefficient, taking 1/3.

2. Standardization:

In order to facilitate the analysis of the diagnosis results, use the following formula to standardize the correlation:

$$
\begin{aligned}
& K^{\prime}\left(N_{i}\right)=\frac{2 \times K\left(N_{i}\right)-K_{\min }-K_{\max }}{K_{\max }-K_{\min }} \\
& \Delta_{i k j l}=\sum_{\delta=1}^{n} \Delta_{i k j l}(\varepsilon) \\
& x_{i}=\sum_{j=1}^{n} \omega_{i j} y_{j}-\theta_{i}
\end{aligned}
$$

3. The transformation formula used in the article is: 
Table 1 Current and voltage detection results when the voltage transformer fails

\begin{tabular}{lllllll}
\hline Num & Current & Voltage & Stability & Direct current & $\begin{array}{l}\text { Voltage } \\
\text { accuracy }\end{array}$ & $\begin{array}{l}\text { High } \\
\text { voltage } \\
\text { output }\end{array}$ \\
\hline 1 & 3.33 & 1 & 3.5 & 3.1 & 3.25 & 3.08 \\
2 & 3 & 0.5 & 4.16 & 4.34 & 3.93 & 4.07 \\
3 & 5.21 & 0.25 & 5.01 & 5.39 & 4.94 & 3 \\
4 & 4 & 0.126 & 3.5 & 5.8 & 3.25 & 3.08 \\
5 & 3 & 0.195 & 4.16 & 6 & 2 & 3.3 \\
6 & 5.21 & 0.092 & 5.01 & 5.39 & 4.94 & 2 \\
\hline
\end{tabular}

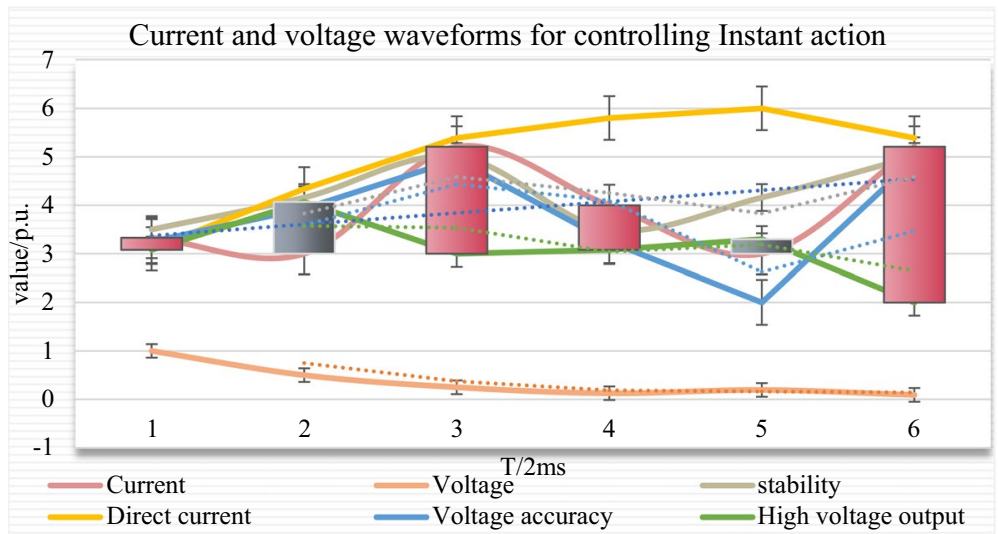

Fig. 2 Current and voltage waveforms for controlling instant action

$$
\begin{aligned}
& \bar{x}_{i}=\frac{x_{i}-x_{\min }}{x_{\max }-x_{\min }} \cdot(\mathrm{HI}-\mathrm{LO})+\mathrm{LO} \\
& y_{i}=f\left(\sum_{j=i}^{k} \omega_{i j} y_{j}-\theta_{i}\right), i \neq j
\end{aligned}
$$

Among them, $x_{i}$ represents the input amount after normalization, which is the input data, $x_{\min }$ is the minimum value and the maximum value in the data, $\mathrm{HI}=0.9, \mathrm{LO}=0.1$, the setting of HI and LO is helpful to reduce the error (Table 1).

\subsection{Experimental results}

1. Current and voltage analysis when the voltage transformer fails

Exploring the role of the IoT diagnostic module in the model on the voltage transformer, the detection results of the current and voltage when the voltage transformer fails are shown in Fig. 2. 
Table 2 Make full use of the acquired data and information results

\begin{tabular}{llllllll}
\hline & Rectifier & C1 & LM7805 & R1-pressure & D-current & I-Things & Regulator \\
\hline Input & 1.96 & 2.17 & 2.45 & 2.08 & 2.08 & 2.35 & 2.45 \\
Output & 2.77 & 2.48 & 2.75 & 2.89 & 2.88 & 2.34 & 2.83 \\
Kernel & 3.33 & 1.3 & 3.5 & 3.1 & 3.25 & 3.08 & 3.06 \\
Regulated & 4.42 & 4.28 & 4.16 & 4.34 & 3.93 & 4.07 & 4.47 \\
Alternating & 5.21 & 5.36 & 5.01 & 5.39 & 4.94 & 5.38 & 4.8 \\
Step-down & 4.84 & 5.34 & 5.45 & 5.45 & 4.94 & 5.24 & 2.3 \\
\hline
\end{tabular}

The relarelationship between step-down module and step-down stability

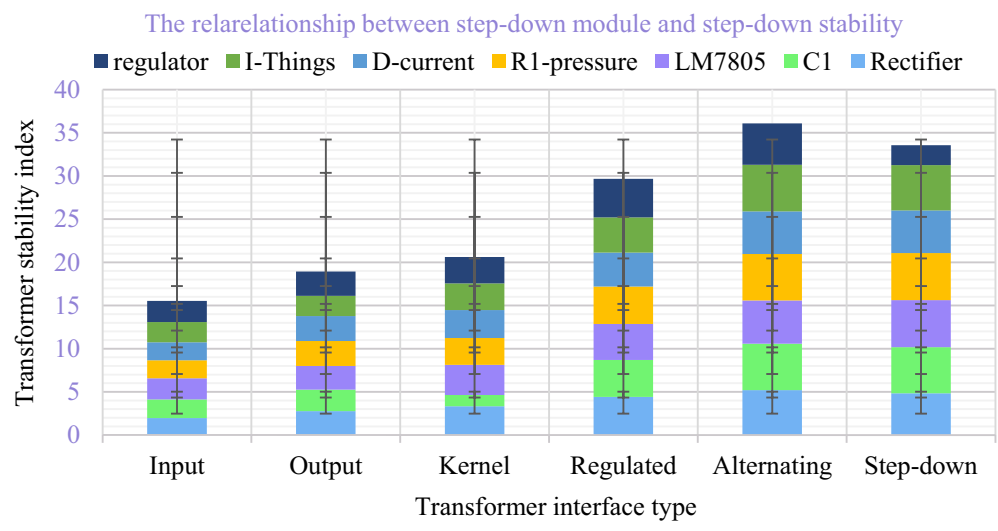

Fig. 3 The relationship between step-down module and step-down stability

It can be seen from Fig. 2 that the average voltage of the transformer sample is not higher than $3 \mathrm{~V}$ every $2 \mathrm{~ms}$, and the fault location also has an impact on the current and voltage after the fault, but the DC line resistance is relatively small, so the fault location has little effect on the short-circuit current and voltage. Therefore, the diagnosis method using the Internet of Things technology comprehensively uses the entropy method to assign weights to each single diagnosis module, which reduces the error interference, and the diagnosis accuracy is much higher than that of the single diagnosis method, which can reflect the amount of uncertainty information of random variables. Use the acquired data information to eliminate the influence of subjective factors and reflect the state of the system more objectively. The system state information is shown in Table 2.

As shown in Fig. 3, because the transformer input and output power supply is $5 \mathrm{~V}$, its core and on-chip peripherals power supply is $3.3 \mathrm{~V}$, so the system uses two sets of regulated power supplies. The $220 \mathrm{~V}$ AC power is first stepped down by a step-down transformer, the step-down AC power is rectified to DC by the rectifier bridge, filtered by $\mathrm{C} 1$, and then entered into the LM7805 voltage regulator module, and finally output $5 \mathrm{~V}$ DC after R1 is divided. As shown in Table 3, the IoT system diagnoses that if the $5 \mathrm{~V}$ DC power is used in the sample and the high voltage output is $5.5 \mathrm{~V}$, the device has characteristics such as low output current, low output voltage accuracy, and lack of stability. 
Table 3 The loT system diagnoses the sample

\begin{tabular}{llllll}
\hline Num & R-deployment & N-communication & loT perception & Node failure & Troubleshooting \\
\hline 1 & 2.08 & 2.1 & 2.37 & 1.86 & 0.3 \\
2 & 2.17 & 2.34 & 2.25 & 0.6 & 2.01 \\
3 & 2.08 & 5 & 2.18 & 1.97 & 1.83 \\
4 & 2.29 & 1.84 & 0.3 & 2.02 & 2.22 \\
5 & 2.3 & 2.6 & 2.41 & 4.3 & 2.82 \\
6 & 3.07 & 3.06 & 3.46 & 3.23 & 1.6 \\
\hline
\end{tabular}

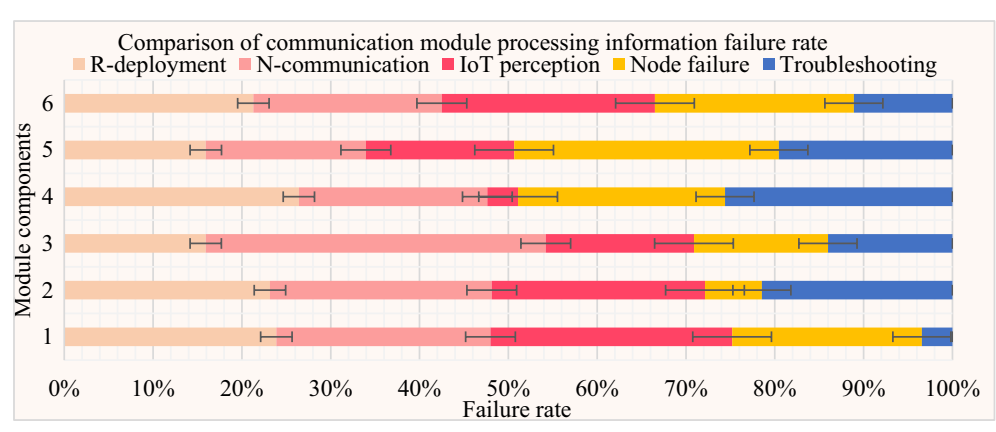

Fig. 4 Comparison of communication module processing information failure rate

Table 4 Reduced a variety of complicated control time

\begin{tabular}{llllll}
\hline Decision & Fault-DM & Matter & Correlation & Diagnosis & $\begin{array}{l}\text { Power } \\
\text { transformer }\end{array}$ \\
\hline 3.28 & 3.86 & 2.32 & 4.86 & 3.02 & 2.47 \\
3.08 & 3.8 & 5.49 & 4.93 & 1.12 & 1.96 \\
3.34 & 4.26 & 5.36 & 4.96 & 7.44 & 2.24 \\
2.91 & 3.88 & 1.02 & 5.33 & 7.15 & 2.04 \\
3.25 & 1.32 & 5.3 & 5.25 & 7.07 & 2.12 \\
\hline
\end{tabular}

As shown in Fig. 4, the joint GPRS communication module and IP module are used to complete the TCP/IP protocol replacement, which in turn completes the receiving and sending of various information, and provides data supply for GPRS; 100 sensing nodes are randomly deployed in an area of $100 \times 100$, and the communication distance of all sensing nodes is set to $50 \mathrm{~m}$, and the fault diagnosis situation when the node failure rate of the wireless sensor network of the sensing layer of the Internet of Things is $0.05 \sim 0.30$ is reduced. This kind of complicated control time, the operation time corresponding to various node failures is shown in Table 4.

As shown in Fig. 5, the decision-level fusion in the research system of this paper uses extension theory to establish a fault diagnosis model, constructs a matter-element matrix and uses correlation functions for evaluation, which can comprehensively diagnose the fault types of power transformers, thereby making data transmission more clear, and the actively transmitted data can directly reach the terminal of the other party's network system after GR47 processing, which greatly reduces the development time of the system. 
Extension theory to build fault diagnosis model

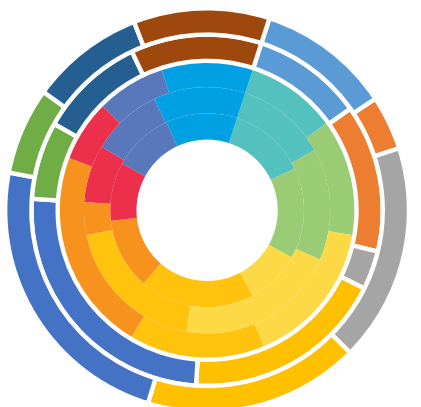

Decision-level fusion

- Fault diagnosis model

Matter element matrix

$\square$ Correlation function

Comprehensive diagnosis

a Power transformer

- Fault type

Data transfer

Fig. 5 Extension theory to build fault diagnosis model

Table 5 The diagnostic effect of the fault diagnosis system model on the transformer

\begin{tabular}{lllllll}
\hline Items & $\mathbf{C H}_{\mathbf{4}}$ & $\mathbf{C}_{\mathbf{2}} \mathbf{H}_{\mathbf{4}}$ & $\mathbf{C}_{\mathbf{2}} \mathbf{H}_{\mathbf{2}}$ & S-transformer & R-bridge & C-filtering \\
\hline 1 & 4.3 & 2.4 & 2 & 2.83 & 2.42 & 2.64 \\
2 & 2.5 & 4.4 & 2 & 3.06 & 2.94 & 3.02 \\
3 & 3.5 & 1.8 & 3 & 4.47 & 4.15 & 4.27 \\
4 & 4.5 & 2.8 & 5 & 4.8 & 5.07 & 4.85 \\
\hline
\end{tabular}

Chromatographic data of transformer in case of fault $\triangle \mathrm{CH} 4 \quad \triangle \mathrm{C} 2 \mathrm{H} 4 \quad \triangle \mathrm{C} 2 \mathrm{H} 2 \quad \approx \mathrm{S}$-transformer $\approx \mathrm{R}$-bridge $\approx \mathrm{C}$-filtering

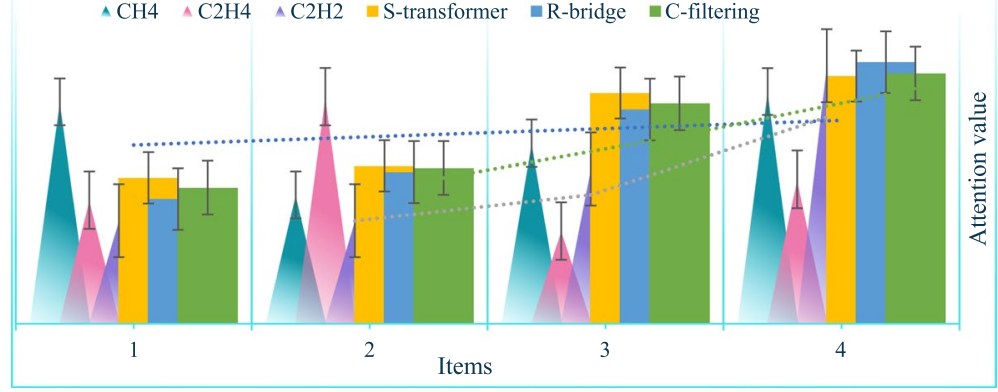

Fig. 6 Chromatographic data of transformer in case of fault

2. Analysis of chromatographic data of transformer in case of failure

Test the diagnostic effect of the transformer fault diagnosis system model on the transformer, analyze the chromatographic data of 10 sets of transformers when the fault occurs, as shown in Table 5.

As shown in Fig. 6, the content of the transformer $\mathrm{C}_{2} \mathrm{H}_{2}$ exceeds the attention value (attention value $\leq 5$ ), an obvious fault occurs inside the transformer, and the fault is characterized as arc discharge. As shown in Table 6, the diagnosis and decision-making results of node module failure types obtained by sensing the symptoms of node failure have high accuracy, and the correct rate of node failure diagnosis is high. In 
Table 6 Redundant test sample data

\begin{tabular}{llllllll}
\hline & Dissolve & Multi-gas & Pheromones & W-data & Change & Volatile & Perception \\
\hline Coefficient & 2.01 & 0.62 & 2.26 & 0.96 & 1.63 & 2.06 & 1.39 \\
Search & 0.6 & 1.17 & 1.5 & 1.36 & 1.61 & 0.62 & 1.92 \\
Troubleshooting & 0.69 & 1.37 & 1.18 & 1.1 & 1.17 & 0.78 & 2.32 \\
Robustness & 2.28 & 2.49 & 2.07 & 2.27 & 2.14 & 2.42 & 1.82 \\
Convergence & 1.96 & 0.37 & 0.88 & 0.96 & 0.68 & 2.36 & 2.05 \\
\hline
\end{tabular}

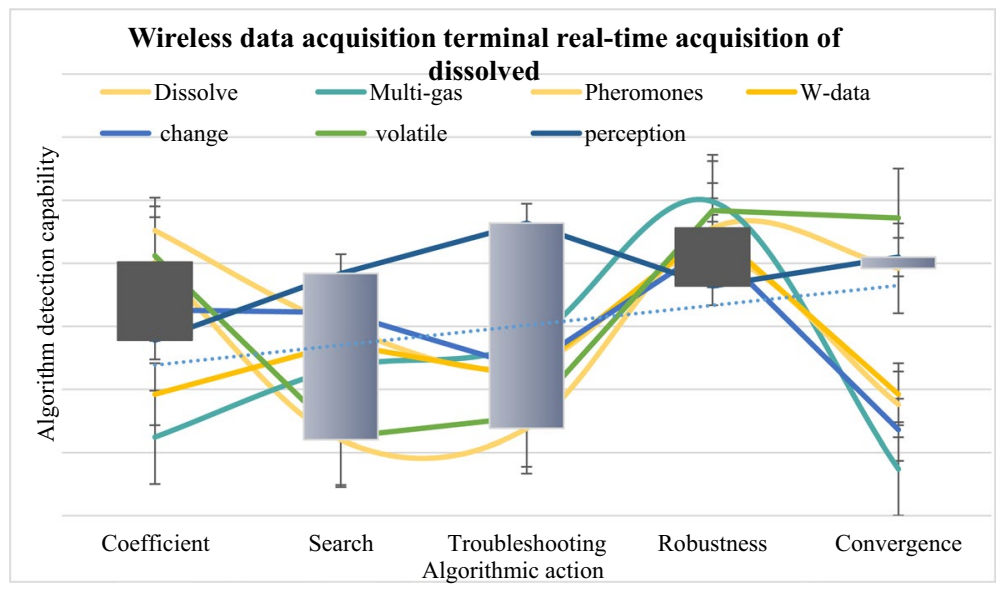

Fig. 7 Wireless data acquisition terminal real-time acquisition of dissolved

Table 7 The composition and content of dissolved gases in transformer oil and severity

\begin{tabular}{llllllll}
\hline Dissolved (\%) & \multicolumn{2}{l}{ Accuracy (\%) } & & \multicolumn{2}{l}{ F1 Index } \\
\cline { 2 - 3 } & $\mathbf{C}_{\mathbf{2}} \mathbf{H}_{\mathbf{2}}$ & $\mathbf{C}_{\mathbf{2}} \mathbf{H}_{\mathbf{4}}$ & $\mathbf{C H}_{\mathbf{4}}$ & & Core clip & Connect & Total \\
\hline 20 & 69.14 & 68.89 & 67.91 & & 0.428 & 0.360 & 0.225 \\
30 & 68.60 & 68.56 & 67.66 & & 0.409 & 0.354 & 0.242 \\
40 & 69.17 & 69.32 & 68.40 & & 0.421 & 0.379 & 0.255 \\
50 & 68.99 & 69.54 & 68.50 & & 0.439 & 0.360 & 0.274 \\
60 & 69.21 & 69.79 & 68.83 & & 0.440 & 0.371 & 0.279 \\
80 & 69.09 & 69.72 & 68.72 & & 0.422 & 0.354 & 0.289 \\
\hline
\end{tabular}

this system, the Internet of Things network has strong self-learning ability, robustness and anti-interference ability, and can determine the redundant test sample data. Therefore, the model constructed in this paper has more excellent characteristics when applied to transformer fault diagnosis.

As shown in Fig. 7, using the Internet of Things technology to sense and obtain these parameters, that is, to collect the multi-component gas dissolved in the transformer oil in real time through the wireless data collection terminal, and analyze the data, pre-process and data fusion, and automatically obtain the transformer the fault diagnosis result can optimize the characteristics and adaptively search the volatile 
Table 8 Calculate the chromatographic data of 10 sets of transformers

\begin{tabular}{lllllll}
\hline Num & $\mathbf{C}_{\mathbf{2}} \mathbf{H}_{\mathbf{2}}$ & $\mathbf{C}_{\mathbf{2}} \mathbf{H}_{\mathbf{4}}$ & $\mathbf{C H}_{\mathbf{4}}$ & Core clip & Connect current & $\begin{array}{l}\text { Total } \\
\text { ground } \\
\text { current }\end{array}$ \\
\hline 1 & & & & & & 7.03 \\
2 & 4.3 & 2.4 & 2 & 7.07 & 7.2 & 2.25 \\
3 & 2.5 & 4.4 & 2 & 2.28 & 2.17 & 2.31 \\
4 & 3.5 & 1.8 & 3 & 2.54 & 2.75 & 2.42 \\
5 & 4.5 & 2.8 & 5 & 2.27 & 2.14 & 4.2 \\
\hline
\end{tabular}

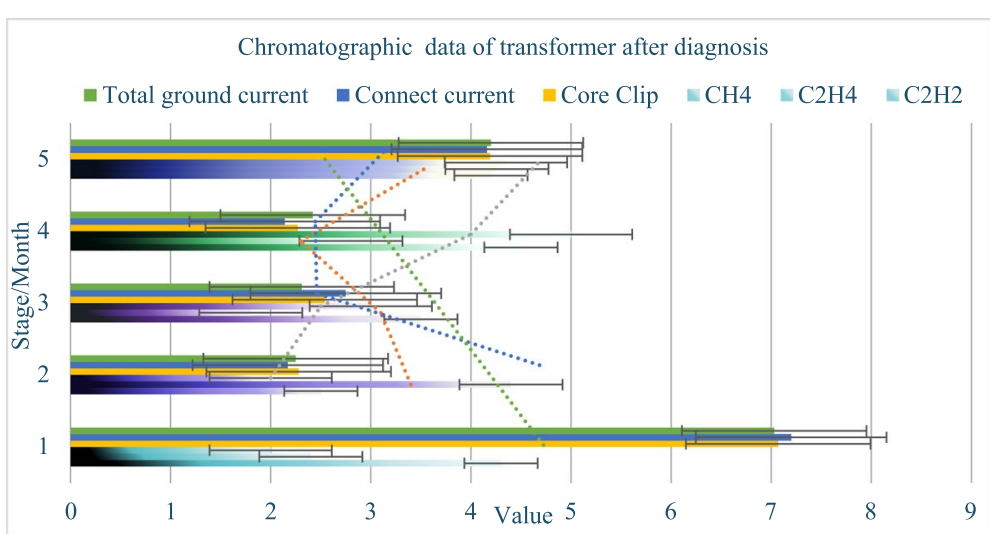

Fig. 8 Chromatographic data of transformer after diagnosis

coefficient to change the pheromone, so that the algorithm detection ability reaches $87 \%$, enhance the robustness of the network and accelerate the convergence.

As shown in Table 7, the composition and content of dissolved gas in transformer oil are closely related to the type of fault and the severity of the fault. The Internet of Things model combines qualitative and quantitative aspects to study the laws and methods for solving contradictory problems, and establish things the matter-element evaluation model of multiple index parameters can completely reflect the comprehensive quality level of things.

\section{Research on the effect of model processing after diagnosis}

To compare and analyze the treatment effect of the fault diagnosis system model on the transformer system diagnosis, the chromatogram data after the diagnosis of 10 sets of transformers are calculated as shown in Table 8.

As shown in Fig. 8, when monitoring the connection current and total ground current of the iron core and clamps in the running state, there is no significant difference between the values and similar transformers, indicating that the discharge defect has been eliminated. The key to transformer fault diagnosis is the determination of fault diagnosis standards. At present, chromatographic analysis of dissolved gases in transformer oil is often used to diagnose latent faults inside transformers. 


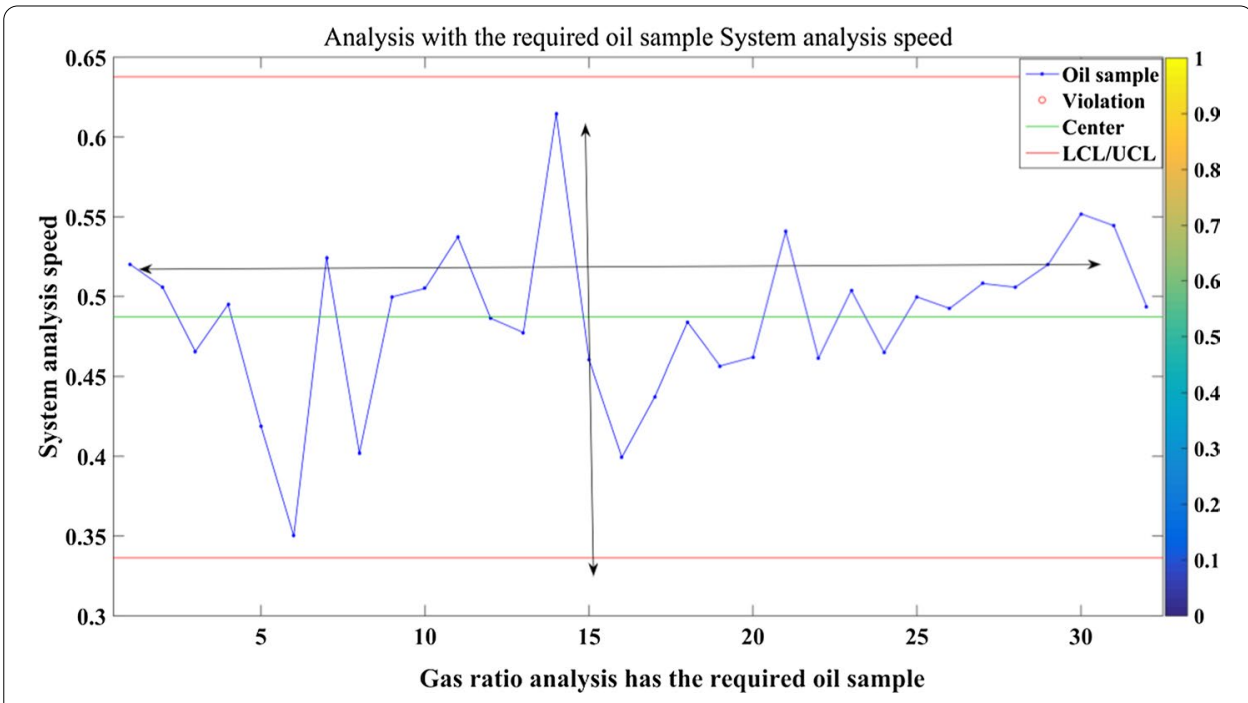

Fig. 9 Analysis with the required oil sample system analysis speed

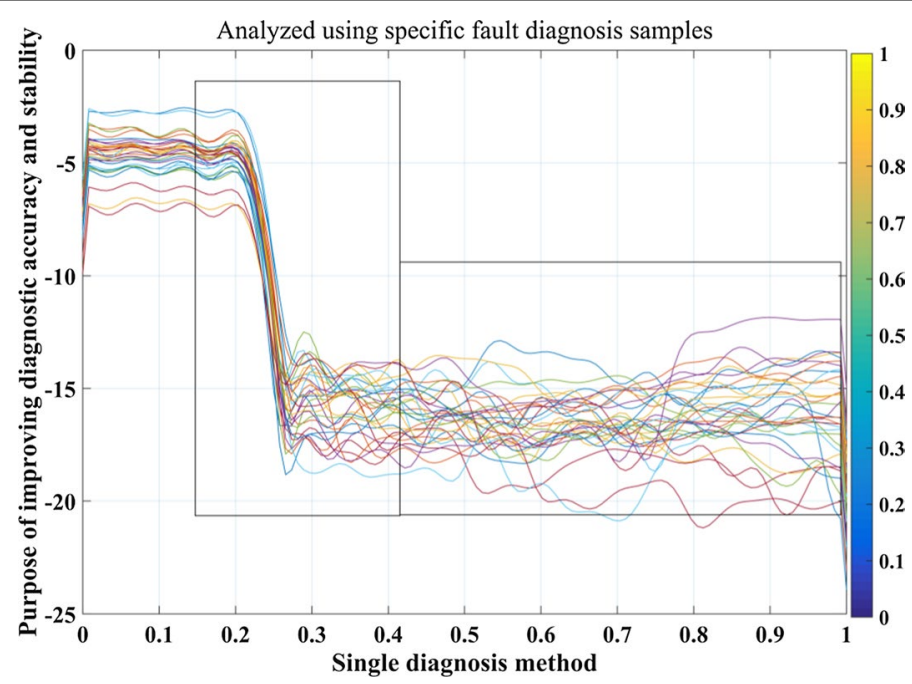

Fig. 10 Analyzed using specific fault diagnosis samples

As shown in Fig. 9, gas ratio analysis has the advantages of fewer oil samples and fast analysis speed, and is an effective method for transformer fault diagnosis. Transformers work on the principle of electromagnetic induction. Therefore, its structure is: two (or more than two) mutually insulated windings are sheathed on a common iron core, and they are connected to each other through the coupling of the magnetic circuit.

As shown in Fig. 10, the paper uses specific fault diagnosis samples for analysis, and the diagnosis accuracy of combining multiple diagnosis methods is better than that of single diagnosis method, which verifies the effectiveness of this method. The use of 


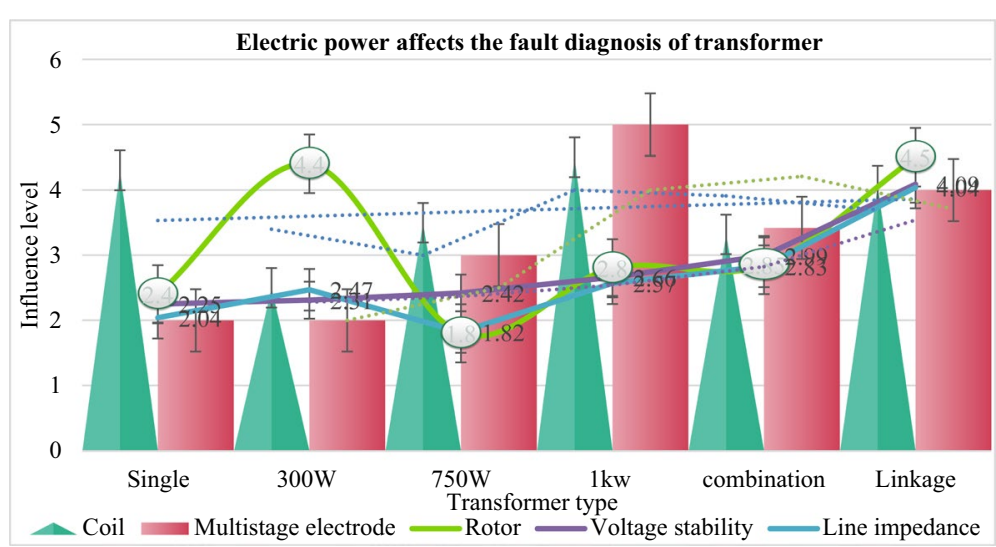

Fig. 11 Electric power affects the fault diagnosis of transformer

Internet of Things diagnostic technology is a new research direction, which can effectively combine different diagnostic methods and ideas, make full use of known information, and achieve the purpose of improving the accuracy and stability of diagnosis.

As shown in Fig. 11, the method in this article is not limited to the fault diagnosis of transformers, but can also be applied to the fault diagnosis of other equipment. The combined diagnosis method is a new method in transformer fault diagnosis, and more in-depth exploration is needed. Further improve the accuracy of diagnosis.

4. Diagnostic analysis of the joint information fusion technology of the Internet of Things

Table 9 Number of training results for verification test set

\begin{tabular}{|c|c|c|c|c|c|c|c|}
\hline Item & Final moisture & Quality & $1 \min (\%)$ & $2 \min (\%)$ & $3 \min (\%)$ & $4 \min (\%)$ & $5 \min (\%)$ \\
\hline N1 & 4.3 & 2.4 & 2 & 4.9 & 5.14 & 4.9 & 5.31 \\
\hline N2 & 2.5 & 4.4 & 2 & 5.35 & 5.17 & 5.36 & 5.47 \\
\hline N3 & 3.5 & 1.8 & 3 & 7.03 & 7.14 & 6.97 & 7 \\
\hline N4 & 4.5 & 2.8 & 5 & 2.25 & 2.04 & 2.3 & 1.99 \\
\hline N5 & 4.91 & 4.95 & 5.11 & 6.87 & 7.07 & 7.2 & 2.17 \\
\hline
\end{tabular}

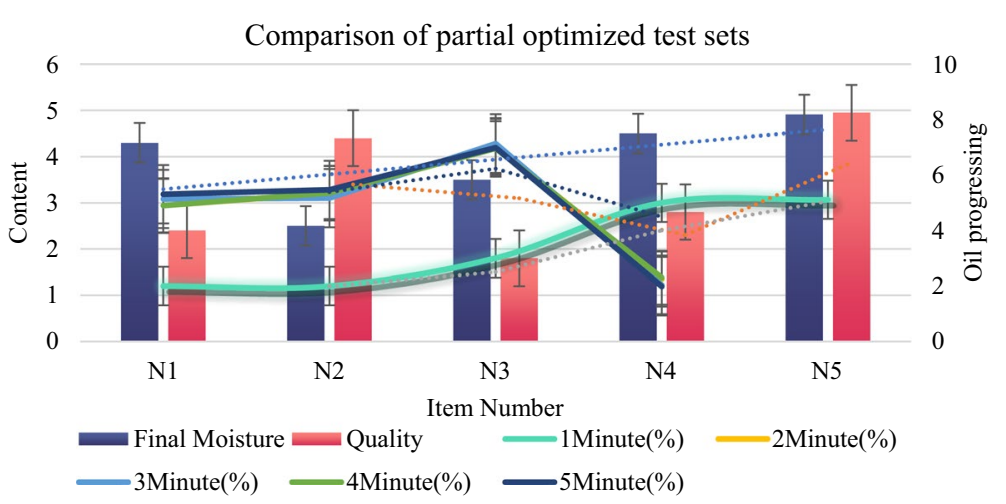

Fig. 12 Comparison of partial optimized test sets 
Table $10 \mathrm{BAI}$ and BMI

\begin{tabular}{llllc}
\hline Item & W-hip & W-h & W-Cir & B-rate \\
\hline BMI & -0.064 & 0.644 & 0.478 & 0.358 \\
BAl & -0.305 & 0.674 & 0.438 & 0.569 \\
Hip ratio & -0.263 & 0.532 & 0.672 & -0.310 \\
Final ratio & -0.154 & 0.413 & 0.910 & 0.28 \\
\hline
\end{tabular}

After training with the training set data, the forward propagation network within the error range is obtained, and then input the test set to verify the training results. The test set data is shown in Table 9.

As shown in Fig. 12, based on the addition of information fusion technology to the Internet of Things, the fault diagnosis system constructed in this article can quickly obtain sample data within 5 Min, which can better cooperate with the terminal data collection link and improve actual efficiency.

As shown in Table 10, the experiment in this article takes the online diagnosis of transformer faults as an example. If a traditional IoT measurement system is used, it will take more than $20 \mathrm{~min}$ to get the final fault parameters. In this way, the required measurement time will be very long. It is long and very wasteful of time and cost. Therefore, the combination of extension theory proposed in this paper is used to train the data samples in the Internet of Things to obtain a network model that meets the convergence conditions and apply it to the data processing server. Through the information fusion technology, the information and data from various information sources are analyzed and processed, and all the fault characteristic quantities of the transformer are used to the greatest extent, and the multi-faceted information of the same object of the transformer is obtained from multiple angles, and the integrated use can achieve more accurate and more scientific online diagnosis. After the initial data evaluation and simple fault diagnosis and analysis, the final result needs to be displayed concisely and clearly on the LCD screen of the monitoring site to facilitate the direct collection of information and management planning. The Internet of Things can reasonably solve the terminal and communication problems in the distribution network. Only by connecting the equipment and accessories in the distribution network to the Internet, the communication work of the distribution network can be completed, and the remote signaling, remote measurement, and automation technology such as remote control. It can be seen that the Internet of Things can deal with the problem of many and frequently changing power distribution terminals.

\section{Training sample statistics}

This paper studies collecting data from one day to many days in the field, selecting one transformer data every hour in the training model, removing invalid measurement point data, selecting $2 / 3$ of them as training samples, and the remaining $1 / 3$ as verification samples. Statistics are shown in Table 11. 
Table 11 Training sample statistics

\begin{tabular}{lccr}
\hline & Normal & Ageing & Fault \\
\hline Number of transformers & 17 & 10 & 10 \\
Sample size & 302 & 150 & 150 \\
Training sample & 203 & 102 & 102 \\
Validation sample & 101 & 53 & 53
\end{tabular}

The influence ratio of each componment structure to the overall diagnosis efficiency

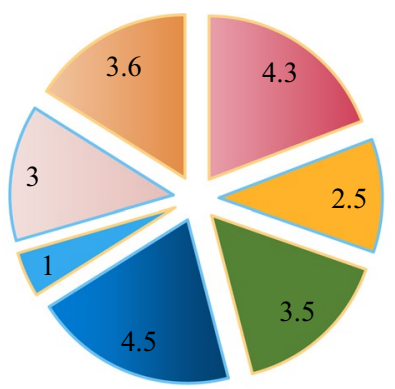

$$
\begin{aligned}
& \square \text { Valid type } \\
& \square \text { Failure type } \\
& \square \text { Diagnosis period } \\
& \square \text { Training set } \\
& =\text { Accurate identification } \\
& \square \text { Network structure } \\
& \square \text { Network parameters }
\end{aligned}
$$

Fig. 13 The influence ratio of each component structure to the overall diagnosis efficiency

Table 12 Data heterogeneity among multiple sensors

\begin{tabular}{llll}
\hline Measurement method & $r$ & $r^{2}$ & SEE \\
\hline BIA & $0.87 / 0.90$ & $0.76 / 0.80$ & $2.22 / 2.01$ \\
ADP & $0.73 / 0.81$ & $0.53 / 0.65$ & $3.99 / 3.45$ \\
DXA & $0.42 / 0.65$ & $0.17 / 0.42$ & $8.18 / 6.86$ \\
\hline
\end{tabular}

It can be seen from Table 11 that the samples in this paper have been screened many times, which effectively reduces the error, and the detection success rate of the power transformer fault diagnosis system model designed in each sample using the Internet of Things is as high as $95.6 \%$. The transformer fault diagnosis method based on the Internet of Things has good pattern recognition capabilities, which can quickly, effectively and accurately diagnose transformer faults, and improve the fault diagnosis capabilities of the entire system through the self-learning function of the network.

As shown in Fig. 13, the network structure and network parameters selected in this paper are reasonable, and the training error is less than 0.0001 , which can accurately identify the failure types of samples in the non-training set. Research on efficient power transformer fault diagnosis methods is of great significance to the safe operation of power systems. In this study, data fusion and extension theory have also been applied to power transformer fault diagnosis.

As shown in Table 12, in the single-sensor system, batch estimation data fusion technology is adopted, which improves the reliability of single-sensor data collection, but does not involve the heterogeneity of data among multiple sensors. Effective processing of the monitoring data of multiple sensors can effectively solve the 
Table 13 Diagnosis results

\begin{tabular}{|c|c|c|c|c|c|}
\hline $\begin{array}{l}\text { Serial } \\
\text { number }\end{array}$ & Fuzzy closeness & $\begin{array}{l}\text { Cause of actual } \\
\text { sample failure }\end{array}$ & Serial number & Fuzzy closeness & $\begin{array}{l}\text { Cause of actual } \\
\text { sample failure }\end{array}$ \\
\hline 1 & 0.976 & $\begin{array}{l}\text { Discharge and over- } \\
\text { heating }\end{array}$ & 6 & 0.932 & $\begin{array}{l}\text { High temperature } \\
\text { overheating }\end{array}$ \\
\hline 2 & 0.965 & $\begin{array}{l}\text { High energy exo- } \\
\text { thermic }\end{array}$ & 7 & 0.923 & $\begin{array}{l}\text { Low temperature } \\
\text { overheating }\end{array}$ \\
\hline 3 & 0.960 & $\begin{array}{l}\text { Temperature over- } \\
\text { heating }\end{array}$ & 8 & 0.865 & Spark discharge \\
\hline 4 & 0.961 & $\begin{array}{l}\text { High energy heat } \\
\text { release }\end{array}$ & 9 & 0.864 & $\begin{array}{l}\text { Low energy discharge } \\
\text { and heat release }\end{array}$ \\
\hline 5 & 0.954 & $\begin{array}{l}\text { High energy dis- } \\
\text { charge }\end{array}$ & 10 & 0.831 & $\begin{array}{l}\text { Medium temperature } \\
\text { overheating }\end{array}$ \\
\hline
\end{tabular}

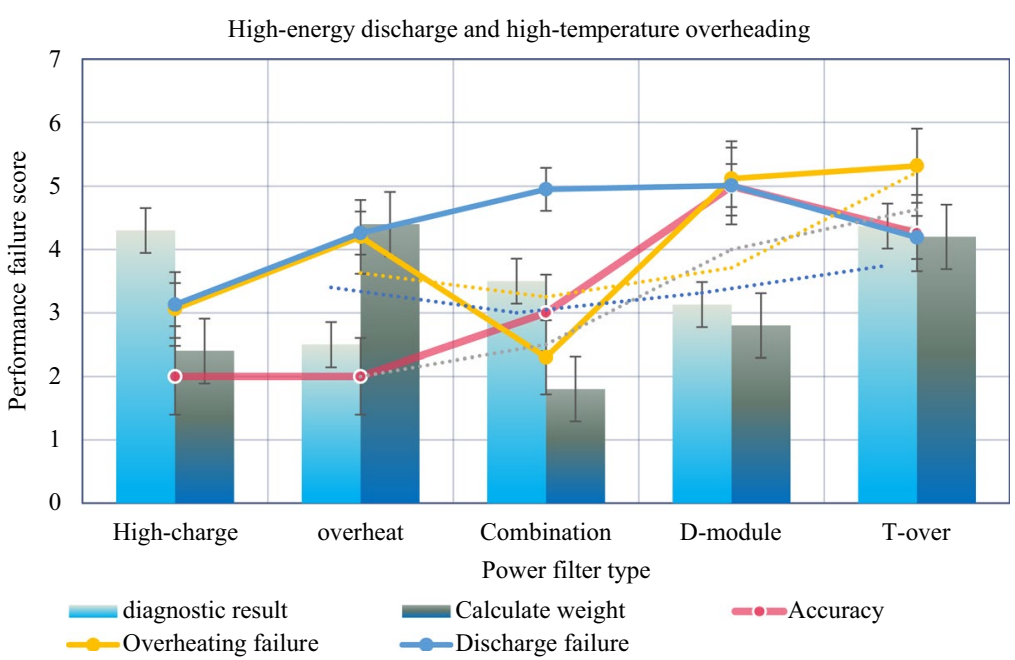

Fig. 14 High-energy discharge and high-temperature overheating

problem that a single sensor cannot diagnose the safe state of the transformer, and the problem that the coding rules are incomplete or there are multiple state types.

6. Exploration of diagnosis results

Quoting the transformer fault diagnosis system model and inputting the fault parameters, the diagnosis results obtained are shown in Table 13.

It can be seen from Table 13 that when most power transformers fail, they are prone to high temperature and overheating. From the diagnosis results, it can be seen that the fault type of the transformer is mostly high-energy discharge, which is consistent with the actual fault type. The transformer is the most critical component in the power system. For low-energy discharge and overheating, it is decomposed into a combination of low-energy discharge and medium-temperature overheating. The main consideration is that the probability of low-energy discharge and medium-temperature overheating simultaneously exists. 
Table 14 Fault type score data table

\begin{tabular}{llllll}
\hline Item & Diagnostic result & $\begin{array}{l}\text { Calculate } \\
\text { weight }\end{array}$ & Accuracy & $\begin{array}{l}\text { Overheating } \\
\text { failure }\end{array}$ & $\begin{array}{l}\text { Discharge } \\
\text { failure }\end{array}$ \\
\hline High-charge & 4.3 & 2.4 & 2 & 3.06 & 3.13 \\
Overheat & 2.5 & 4.4 & 2 & 4.2 & 4.26 \\
Combination & 3.5 & 1.8 & 3 & 2.3 & 4.95 \\
D-module & 3.13 & 2.8 & 5 & 5.12 & 5.01 \\
T-over & 4.37 & 4.2 & 4.26 & 5.32 & 4.19 \\
\hline
\end{tabular}

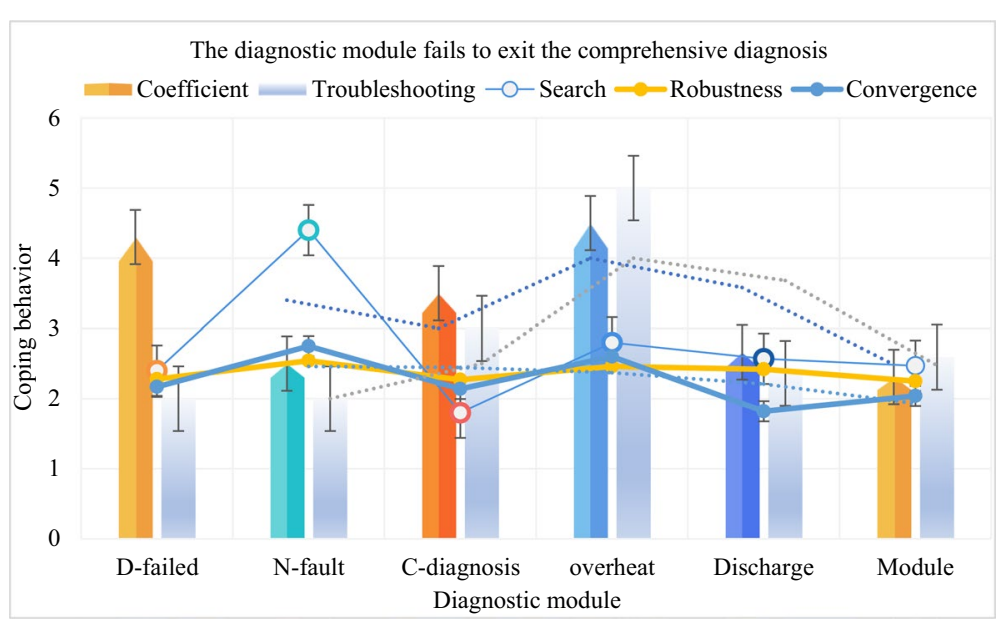

Fig. 15 The diagnostic module fails to exit the comprehensive diagnosis

Table 15 False judgment rate of fault type

\begin{tabular}{llllll}
\hline & Coefficient & Search & Troubleshooting & Robustness & Convergence \\
\hline D-failed & 4.3 & 2.4 & 2 & 2.28 & 2.17 \\
N-fault & 2.5 & 4.4 & 2 & 2.54 & 2.75 \\
C-diagnosis & 3.5 & 1.8 & 3 & 2.27 & 2.14 \\
Overheat & 4.5 & 2.8 & 5 & 2.46 & 2.6 \\
Discharge & 2.66 & 2.57 & 2.36 & 2.42 & 1.82 \\
Module & 2.31 & 2.47 & 2.59 & 2.25 & 2.04 \\
\hline
\end{tabular}

As shown in Fig. 14, for the same consideration, high-energy discharge and overheating are decomposed into a combination of high-energy discharge and high-temperature overheating. The implementation process of the data module involved in the diagnosis in this system is: decompose each diagnosis result, calculate the weighted (accuracy) scores of overheating faults and discharge faults, score according to the principle of rounding, and then judge according to the size of the score at the level, the fault type is finally obtained, and the score is shown in Table 14. 
As shown in Fig. 15, if a diagnosis module fails to diagnose (no diagnosis result) or diagnoses no fault, the diagnosis module will automatically exit the comprehensive diagnosis. In this way, it is easy for two modules to think that there is discharge (or overheating), and only one module thinks that there is both overheating (and discharge), and the diagnosis result will be a discharge and overheating fault. This is mainly considered from the aspect of causing users to pay enough attention.

As shown in Table 15, using the combined diagnosis model to diagnose transformer faults, the misjudgment rate of each fault type is much lower than that of using a single diagnostic module. It can be seen that the use of the Internet of Things and a series of methods to diagnose power transformer faults is better than traditional diagnostic methods, and can effectively improve the accuracy of fault diagnosis.

\section{Conclusion}

This paper mainly expounds the combination of Internet of Things and smart grid technology and the role of Internet of Things technology in power maintenance, analyzes the use of power transformer and its fault diagnosis system, and focuses on GPRS communication module and fault diagnosis module in data acquisition terminal, and deeply explores transformer fault diagnosis and data management subsystem based on information fusion technology. It can be seen that GPRS communication network combined with Internet of Things technology can effectively diagnose the fault system of power transformer.

The research results of this paper: 30 groups of transformer fault samples are selected, and 10 groups are randomly selected for network training, and the remaining samples are used for testing. The transformer fault system diagnosis model is established for each group of power transformers for four consecutive months. This paper studies the detection success rate of power transformer fault diagnosis system model based on the Internet of Things. The training error is less than 0.0001, and it can accurately identify the fault types of non training samples.

Although many scholars have made outstanding achievements in the research of power transformer fault diagnosis system and Internet of Things technology, but at the same time, in view of some limitations of this study, we hope that the Internet of Things and transformer fault diagnosis technology should also consider other problems that are easy to be ignored while meeting the demand, so as to better improve the fault diagnosis technology and more widely use it. If the fault system diagnosis technology can be further improved and applied to power transformer better, it will greatly improve the safety of people's life.

\section{Abbreviations}

PLC: Programmable Logic Controller; SNR: Signal Noise Ratio; IOT: Indexed Organized Table; TCP: Transmission Control Protocol; IP: Internet Protocol; LCD: Liquid crystal display; DC: Direct current; GPRS: General Packet Radio Service.

Acknowledgements

The authors thank the editor and anonymous reviewers for their helpful comments and valuable suggestions.

\section{Author's contributions}

All authors take part in the discussion of the work described in this paper. All authors read and approved the final manuscript.

Funding

This work was supported by Digital grid platform (Hainan) construction project (number: 072900HQ42190001). 
Consent for publication.

Approved.

\section{Competing interests}

There is no potential competitive advantage in our paper. All the authors have reviewed the manuscript and agreed to submit it to your magazine. We confirm that the contents of the manuscript have not been published or submitted for publication elsewhere.

\section{Author details}

${ }^{1}$ Hainan Power Grid Co., Ltd, Haikou 460100, Hainan, China. ${ }^{2}$ Hainan Power Grid Co., Ltd., Information Communication Branch, Haikou 460100, Hainan, China.

Received: 2 September 2020 Accepted: 2 December 2020

Published online: 05 February 2021

\section{References}

1. M. Di Renzo, W. Lu, System-level analysis/optimization of cellular networks with simultaneous wireless information and power transfer: stochastic geometry modeling. IEEE Trans. Veh. Technol. 66(3), 1-1 (2017)

2. L. Juanli, X. Jiacheng, Y. Zhaojian et al., Fault diagnosis method for a mine hoist in the internet of things environment. Sensors 18(6), 1920 (2018)

3. Y. Qian, J. Yan, H. Guan et al., Design of hybrid wireless and power line sensor networks with dual-interface relay in IOT. IEEE Internet Things J. 6(1), 239-249 (2019)

4. Y.B. Zikria, H. Yu, M.K. Afzal et al., Internet of Things (loT): operating system, applications and protocols design, and validation techniques. Future Gen. Comput. Syst. 88, 699-706 (2018)

5. A.M. Zungeru, S. Subashini, P. Vetrivelan, NR-DCSK-based MIMO chaotic communication system: chapter 22, in Wireless Communication Networks and Internet of Things. Lecture Notes in Electrical Engineering, vol. 493 (2019), pp. 207-215. 10.1007/978-981-10-8663-2

6. A.M. Zungeru, S. Subashini, P. Vetrivelan, Throughput analysis of MacroUE for varied transmit power of small cell in heterogeneous network: chapter 17, in Wireless Communication Networks and Internet of Things. Lecture Notes in Electrical Engineering, vol. 493 (2019), pp. 161-170. 10.1007/978-981-10-8663-2

7. G. Manogaran, N. Chilamkurti, C.H. Hsu, Emerging trends, issues, and challenges in Internet of Medical Things and wireless networks. Pers. Ubiquitous Comput. 22(5-6), 879-882 (2018)

8. M.S. Mahmoud, A.A.H. Mohamad, A study of efficient power consumption wireless communication techniques/ modules for Internet of Things (IoT) applications. Adv. Internet Things 06(2), 19-29 (2016)

9. S. Krueger, Critical theory of communication: new readings of Lukács, Adorno, Marcuse, Honneth and Habermas in the age of the internet. Inf. Commun. Soc. 21(11-12), 1808-1812 (2018)

10. A.M. Zungeru, S. Subashini, P. Vetrivelan, Dependency analysis of control parameter configuration on ISD and random mobility of UE in LTE-A network: chapter 16, in Wireless Communication Networks and Internet of Things. Lecture Notes in Electrical Engineering, vol. 493 (2019), pp. 151-159. 10.1007/978-981-10-8663-2

11. Z. Ma, M. Xiao, Y. Xiao et al., High-reliability and low-latency wireless communication for Internet of Things: challenges, fundamentals and enabling technologies. IEEE Internet Things J. 6, 7946-7970 (2019)

12. A.M. Zungeru, S. Subashini, P. Vetrivelan, Modelling and performance analysis of Wi-fi offloading: chapter 4, in Wireless Communication Networks and Internet of Things. Lecture Notes in Electrical Engineering, vol. 493 (2019), pp. 33-39. 10.1007/978-981-10-8663-2

13. A.M. Zungeru, S. Subashini, P. Vetrivelan, Enhancement of QOS parameters in cluster-based wireless sensor network using cooperative MIMO: chapter 20, in Wireless Communication Networks and Internet of Things. Lecture Notes in Electrical Engineering, vol. 493 (2019), pp. 187-195. 10.1007/978-981-10-8663-2

14. K.V. Arya, R.S. Bhadoria, N.S. Chaudhari, Realizing the wireless technology in Internet of Things (IoT): chapter 10, in Emerging Wireless Communication and Network Technologies (2018), pp. 173-192. https://doi. org/10.1007/978-981-13-0396-8

15. M.M. Rana, W. Xiang, loT communications network for wireless power transfer system state estimation and stabilization. IEEE Internet Things J. 5, 4142-4150 (2018)

16. A. Vinel, Chen W SE, Xiong N N, et al. Enabling wireless communication and networking technologies for the internet of things [Guest editorial]. IEEE Wirel. Commun. 2016, 23(5):8-9.

17. A.M. Zungeru, S. Subashini, P. Vetrivelan, Efficient relaying for enhanced network longevity for E-health IOT services in medical body area networks: chapter 2, in Wireless Communication Networks and Internet of Things, Lecture Notes in Electrical Engineering, vol. 493 (2019), pp. 13-19. 10.1007/978-981-10-8663-2

18. P. Ramezani, A. Jamalipour, Toward the evolution of wireless powered communication networks for the future Internet of Things. IEEE Netw. 31(6), 62-69 (2017)

19. W. Ejaz, A. Anpalagan, M.A. Imran et al., Internet of Things (IoT) in $5 \mathrm{G}$ wireless communications. IEEE Access 4 , 10310-10314 (2017)

20. Z. Junqing, D. Trung, W. Roger et al., Securing wireless communications of the Internet of Things from the physical layer: an overview. Entropy 19(8), 420 (2017)

21. S. Gao, G. Tian, X. Dai et al., A novel distributed linear-spatial-array sensing system based on multichannel LPWAN for large-scale blast wave monitoring. IEEE Internet Things J. 6(6), 9679-9688 (2019)

22. V. Freschi, E. Lattanzi, A study on the impact of packet length on communication in low power wireless sensor networks under interference. IEEE Internet Things J. 6, 3820-3830 (2019)

23. O. Kaiwartya, A.H. Abdullah, Y. Cao et al., Virtualization in wireless sensor networks: fault tolerant embedding for Internet of Things. IEEE Internet Things J. 5(2), 571-580 (2018) 
24. P. Hu, J. Zhang, 5G-enabled fault detection and diagnostics: how do we achieve efficiency? IEEE Internet Things J. 7(4), 3267-3281 (2020)

25. G. Yang, D. Yuan, Y.C. Liang et al., Optimal resource allocation in full-duplex ambient backscatter communication networks for wireless-powered loT. IEEE Internet Things J. 6, 2612-2625 (2018)

\section{Publisher's Note}

Springer Nature remains neutral with regard to jurisdictional claims in published maps and institutional affiliations.

Submit your manuscript to a SpringerOpen ${ }^{\circ}$ journal and benefit from:

- Convenient online submission

- Rigorous peer review

- Open access: articles freely available online

- High visibility within the field

Retaining the copyright to your article

Submit your next manuscript at $\mathbf{s p r i n g e r o p e n . c o m ~}$ 